\title{
Challenges of Agile Development: A Cause-and-Effect Analysis
}

\author{
Tobias Sebastian Schmidt and Kristin Paetzold
}

\begin{abstract}
Agile development as an alternative to traditional plan-driven approaches gains rising popularity in both software and non-software industries due to its advantages in dynamic and uncertain environments. Although its implementation challenges are widely explored, interdependencies between them are mostly neglected in recent papers. Practice and academia, therefore, often try to find local optimizations without (a) considering the interdependencies and (b) differentiating between causes and effects. By using the network theory this investigation sets up a directed network containing 241 challenges (nodes) and 360 dependencies (edges) and executes a cause-and-effect analysis. To identify challenges that are most crucial and, thus, of highest importance for future research, the analysis takes each challenges' (i) degree of being a cause, (ii) impact and (iii) range of influence into account. 'Granting freedom of action and decision', 'integrating agile methods in traditional organizations' and 'composing agile teams' turn out to be the top three challenges.
\end{abstract}

\footnotetext{
T.S. Schmidt (四)

Institute for Innovation and Technology Management, Werner-Heisenberg-Weg 39, 85577 Neubiberg, Germany e-mail: tobias.schmidt@unibw.de

K. Paetzold Institute for Technical Product Development, Werner-Heisenberg-Weg 39, 85577 Neubiberg, Germany e-mail: kristin.paetzold@unibw.de 